\title{
Long-Term Potentiation in the Neonatal Rat Barrel Cortex In Vivo
}

\author{
Shuming An, Jenq-Wei Yang, Haiyan Sun, Werner Kilb, and Heiko J. Luhmann \\ Institute of Physiology and Pathophysiology, University Medical Center of the Johannes Gutenberg University, D-55128 Mainz, Germany
}

Long-term potentiation (LTP) is important for the activity-dependent formation of early cortical circuits. In the neonatal rodent barrel cortex, LTP has been studied only in vitro. We combined voltage-sensitive dye imaging with extracellular multielectrode recordings to study whisker stimulation-induced LTP in the whisker-to-barrel cortex pathway of the neonatal rat barrel cortex in vivo. Single whisker stimulation at $2 \mathrm{~Hz}$ for $10 \mathrm{~min}$ induced an age-dependent expression of LTP in postnatal day (P) 0 to P14 rats, with the strongest expression of LTP at P3-P5. The magnitude of LTP was largest in the activated barrel-related column, smaller in the surrounding septal region, and no LTP could be observed in the neighboring barrel. Current source density analyses revealed an LTP-associated increase of synaptic current sinks in layer IV/lower layer II/III at P3-P5 and in the cortical plate/upper layer V at P0-P1. Our study demonstrates for the first time an age-dependent and spatially confined LTP in the barrel cortex of the newborn rat in vivo.

\section{Introduction}

Long-term potentiation (LTP) is important for the activitydependent development and refinement of early neuronal circuits (Feldman et al., 1999; Daw et al., 2007; Inan and Crair, 2007). Previous studies in thalamocortical slices of newborn rats have demonstrated that pairing presynaptic afferent stimulation with postsynaptic depolarization of layer IV cells induces a significant LTP, which gradually decreases in its magnitude during the critical period between postnatal day (P) 3 and P7 (Crair and Malenka, 1995; Isaac et al., 1997; Barth and Malenka, 2001). This developmental period coincides with the formation of the cortical barrel map (Fox, 1992; Fox et al., 1996; Foeller and Feldman, 2004).

Whether LTP can be induced by whisker stimulation in the adult barrel cortex in vivo is a matter of debate. Multiwhisker stimulation by air puffs at $5 \mathrm{~Hz}$ for $30 \mathrm{~s}$ did not induce LTP in adolescent mice (Takata et al., 2011), while multiwhisker stimulation at 2 or $8 \mathrm{~Hz}$ for $10 \mathrm{~min}$ induced a stable LTP in layers II/III and IV of the barrel cortex of mature mice (Mégevand et al., 2009). Whether LTP can be elicited in the neocortex in vivo before the onset of the critical period has not been investigated yet. Therefore, we studied the question of whether single-whisker stimulation may elicit LTP in the immature (P0-P14) rat barrel cortex in vivo. In addition, we addressed the following three questions: (1) whether the expression of LTP reveals any agedependence during the first two postnatal weeks, (2) if the LTP is

\footnotetext{
Received March 12, 2012; revised April 30, 2012; accepted May 17, 2012.

Author contributions:S.A., J.-W.Y., W.K., and H.J.L. designed research; S.A. and J.-W.Y. performed research;S.A., J.-W.Y., H.S., W.K., and H.J.L. analyzed data; S.A., J.-W.Y., H.S., W.K., and H.J.L. wrote the paper.

This work was supported by the Deutsche Forschungsgemeinschaft (DFG FOR 1341, BaCOFun). We thank Beate Krumm for excellent technical assistance.

The authors have declared that no conflict of interest exists.

Correspondence should be addressed to Heiko J. Luhmann, Institute of Physiology and Pathophysiology, University Medical Center Mainz, Duesbergweg 6, D-55128 Mainz, Germany. E-mail: luhmann@uni-mainz.de.

DOI:10.1523/JNEUROSCI.1212-12.2012

Copyright $\odot 2012$ the authors $\quad 0270-6474 / 12 / 329511-06 \$ 15.00 / 0$
}

restricted to the stimulated barrel-related column, and (3) which cortical layers reveal LTP. To address these questions, we performed multichannel extracellular electrophysiological recordings from barrels and barrel-related columns that were functionally identified by voltage-sensitive dye responses following single-whisker stimulation. Using this approach, we found that physiological whisker stimulation ( $2 \mathrm{~Hz}$ for $10 \mathrm{~min}$ ) induces an age-dependent and spatially confined LTP of the whisker-tobarrel cortex pathway in the neonatal rat barrel cortex in vivo.

\section{Materials and Methods}

Surgical preparation. All experiments were performed in accordance with the national laws for the use of animals in research and approved by the local ethical committee (\#23177-07/G10-1-010). Voltage-sensitive dye imaging (VSDI) and field potential (FP) recordings were made in the barrel cortex of head-restrained neonatal Wistar rats (either sex) aged $\mathrm{P} 0-\mathrm{P} 14$. Surgical preparation and electrophysiological recordings were as described previously (Yang et al., 2009). Anesthesia was induced by initial hypothermia and intraperitoneal urethane injection $(0.5-1 \mathrm{~g} / \mathrm{kg}$; Sigma-Aldrich). The head of the pup was fixed into the stereotaxic apparatus. The skull above the barrel cortex was carefully removed leaving the dura mater intact. During recording, the body was kept at a constant temperature of $37^{\circ} \mathrm{C}$ by a heating blanket.

Whisker stimulation. All whiskers except B2, C2, and D2 were trimmed under anesthesia following the surgical preparation. A single whisker (usually C2) was stimulated $\sim 1 \mathrm{~mm}$ from the snout using a miniature solenoid actuator ([odified from Krupa et al. (2001)] that generated for $32 \mathrm{~ms}$ a deflection in the rostral-to-caudal direction at $136 \mathrm{~mm} / \mathrm{s}\left(4^{\circ} / \mathrm{ms}\right)$. Recording sessions consisted of a $30 \mathrm{~min}$ baseline recording period, followed by a $10 \mathrm{~min} 2 \mathrm{~Hz}$ stimulation period for the induction of LTP and a 60 min poststimulation period (Fig. $1 \mathrm{~A}$ ). During the baseline recording and poststimulation period, two stimuli with a $60 \mathrm{~s}$ interval were applied every $5 \mathrm{~min}$.

Voltage-sensitive dye imaging. Functional barrel-related columns were identified by VSDI as described in detail previously (Berger et al., 2007). The voltage-sensitive dye RH1691 (Optical Imaging) was dissolved in a saline solution containing the following (in $\mathrm{mm}$ ): $125 \mathrm{NaCl}, 2.5 \mathrm{KCl}, 10$ HEPES ( $\mathrm{pH} 7.3$ with $\mathrm{NaOH}$ ); and was topically applied to the exposed 
cortical surface for $15 \mathrm{~min}$. Excitation light from a red LED (MRLED $625 \mathrm{~nm}$; Thorlabs) was bandpass filtered $(630 / 30 \mathrm{~nm})$, reflected toward the sample by a $650 \mathrm{~nm}$ dichroic mirror, and focused by a $25 \mathrm{~mm}$ Navitar video lens (Stemmer Imaging). Emitted fluorescence was collected via the same optical pathway, longpass filtered $(660 \mathrm{~nm})$, and focused via another $25 \mathrm{~mm}$ Navitar lens and a C-mount extension tube onto the chip of a MiCam Ultima L high speed camera (Scimedia). Every pixel of this camera collected light from a cortical region of $26 \times 26 \mu \mathrm{m}^{2}$.

Multielectrode recordings. As described previously (Yang et al., 2009), FPs were recorded with a four-shank or one-shank 16-channel Michigan electrode (1-2 M $\Omega$; NeuroNexus Technologies). On the four-shank electrode, the recording sites were separated by $125 \mu \mathrm{m}$ in horizontal direction and $50 \mu \mathrm{m}$ in vertical direction. On the one-shank electrode, the recording sites were separated by $100 \mu \mathrm{m}$ for experiments performed in $\mathrm{P} 3-\mathrm{P} 5$ rats and 50 $\mu \mathrm{m}$ for $\mathrm{P} 0-\mathrm{P} 1$ rats. For FP recordings, electrodes were positioned perpendicular to the cortical layers with at least one shank inserted into the center of the activated barrelrelated column, as identified with VSDI (Fig. $1 A)$. Electrodes were labeled with DiI $\left(1,1^{\prime}\right.$ dioctadecyl-3,3,3',3'-tetramethyl indocarbocyanine; Invitrogen) for subsequent histological reconstruction of the electrode tracks in Nisslstained coronal sections through the barrel cortex (see Fig. 3A). FPs were recorded for at least $2 \mathrm{~h}$ at a sampling rate of $20 \mathrm{kHz}$ using a multichannel extracellular amplifier and $\mathrm{MC}$ RACK software (Multi Channel Systems). Current source density (CSD) profiles were calculated from the FP profiles according to a three-point formula described by Freeman and Nicholson (1975). The CSD values $I_{\mathrm{m}}$ were derived from the second spatial deviation of the extracellular FPs $\Phi$ and calculated by the finite-difference formula $\mathrm{I}_{\mathrm{m}}=-\left(1 / k h^{2}\right) \sum_{m=-n}^{n} a_{m} \Phi(X+m h)$, in which $h$ gives the distance between two successive recording points and $X$ is the coordinate perpendicular to the cortical layer. The remaining constants are as follows: $n=1, k=1, a_{0}=-2$, and $a_{ \pm 1}=1$.

The efficacy of synaptic transmission was calculated from the slope of the initial negativation of the evoked FP response. If not stated otherwise, we analyzed the FP from the electrode located in the cortical plate (at $\mathrm{P} 0-\mathrm{P} 1$ ) or in layer IV (P3-P14), which showed the largest FP response. Evoked FP responses were visually inspected to exclude artifacts or contaminations with spontaneous activity. The slope was determined between $20 \%$ and $80 \%$ of maximal FP amplitude and was normalized to the average slope recorded during a $30 \mathrm{~min}$ baseline interval. For statistical analyses, slope values were averaged for 35-60 min poststimulus intervals. Data analysis was performed with MATLAB software version R2008B.

Histology. After electrophysiological recordings, animals were deeply anesthetized by hypothermia and decapitated. The skull was removed and a section including the cortical barrel field was cut tangential to the cortical surface. The section was fixed in $4 \%$ paraformaldehyde for $24 \mathrm{~h}$

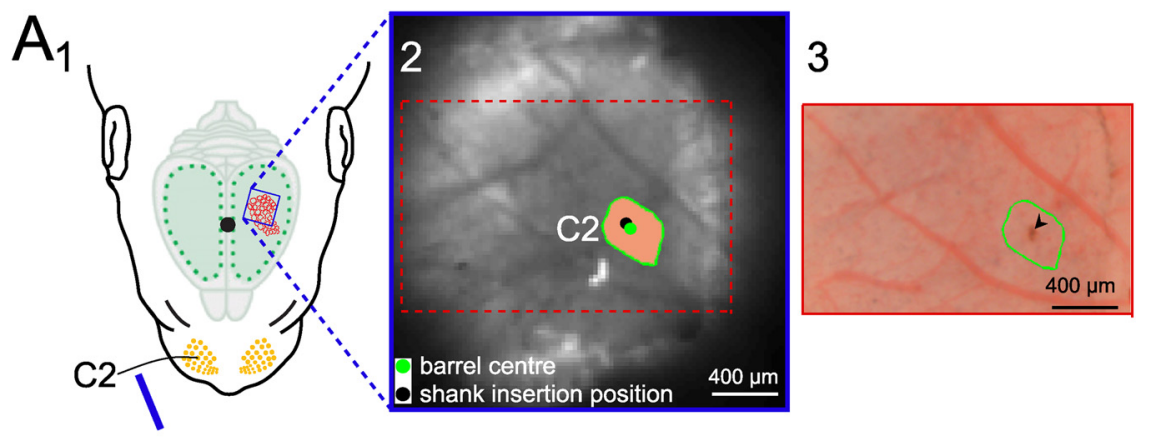

single whisker stimulus
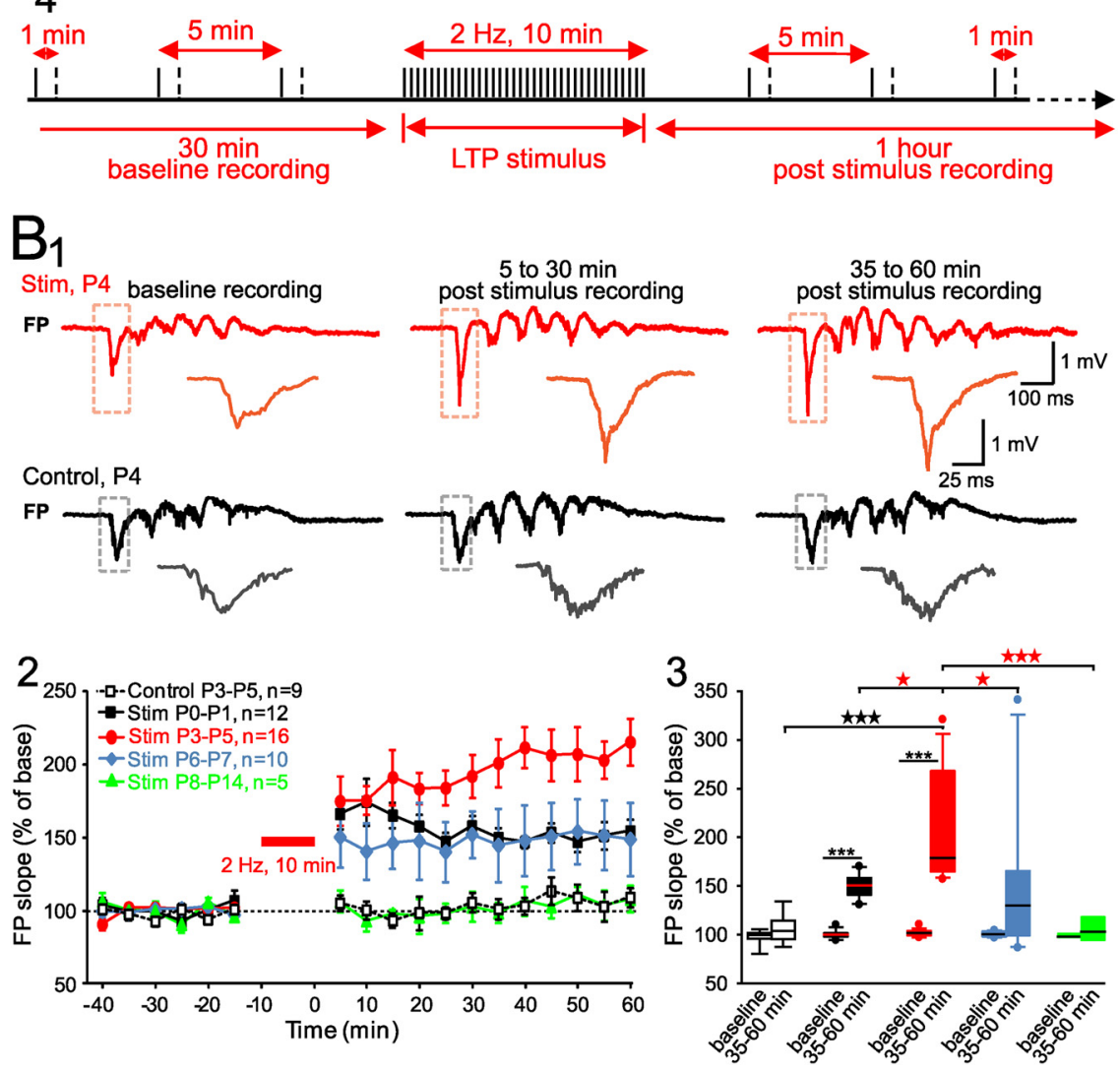

Figure 1. Mechanical deflection of a single whisker for $10 \mathrm{~min}$ at $2 \mathrm{~Hz}$ elicits LTP in barrel cortex of newborn rats in vivo. $\boldsymbol{A}_{\text {, }}$ Schematic diagram of the experimental setup illustrating selective mechanical stimulation of the $C 2$ whisker $\left(A_{1}\right)$ and simultaneous VSDI in the barrel cortex $\left(\boldsymbol{A}_{2}\right)$. The exposed barrel cortex was stained with the voltage-sensitive dye RH1691. A single-whisker deflection of the $\mathrm{C} 2$ whisker elicits a local VSDI response in a P3 rat. The green dot indicates the center of the $\mathrm{C} 2$ barrel-related cortical column. The black dot is the electrode insertion position. Orange represents the region of the $\mathrm{C} 2$ whisker stimulationevoked VSDI response. $\boldsymbol{A}_{3}$ shows the same area after termination of the electrophysiological recording and retraction of the recording electrode. The red dot and arrowhead show the electrode insertion point. $A_{4}$, Stimulation protocol for induction of LTP. During baseline recording, the whisker was deflected twice per $5 \mathrm{~min}$ at $1 \mathrm{~min}$ intervals for $30 \mathrm{~min}$. For LTP induction, the whisker was deflected at a frequency of $2 \mathrm{~Hz}$ for $10 \mathrm{~min}$. Afterward, the same two stimuli per $5 \mathrm{~min}$ were used again for $60 \mathrm{~min}$ during the poststimulation recording period. $\boldsymbol{B}$, Time course of $F$ P responses before and after induction of LTP. $\boldsymbol{B}_{1}$, Representative FP recording during baseline and the 5-30 and 35- 60 min phases after $2 \mathrm{~Hz}$ stimulation (red) or without $2 \mathrm{~Hz}$ stimulation (black) in a $\mathrm{P} 4$ rat. $\boldsymbol{B}_{2 \text {, }}$ Relative FP slopes recorded in different age groups and in P3-P5 control group. Data are expressed as mean \pm SEM. $\boldsymbol{B}_{3}$, Box plots of FP slopes in P3-P5 control and LTP groups of different ages with baseline and 35-60 min poststimulation.

and washed in $0.01 \mathrm{~m}$ PBS. Serial 200- $\mu \mathrm{m}$-thick tangential slices of flattened barrel cortex were cut on a cryotome and stained for serotonin transporter (5-HTT) using a modification of a previously published protocol (Piñon et al., 2009). Slices were washed with PBS, followed by blocking and permeabilization with $7 \%$ normal goat serum and $0.8 \%$ Triton in PBS [ $2 \mathrm{~h}$ at room temperature (RT)] and incubated overnight at RT in rabbit anti-serotonin transporter polyclonal antibody (1:1000, AB9726; Millipore Bioscience Research Reagents). Antibody staining was 

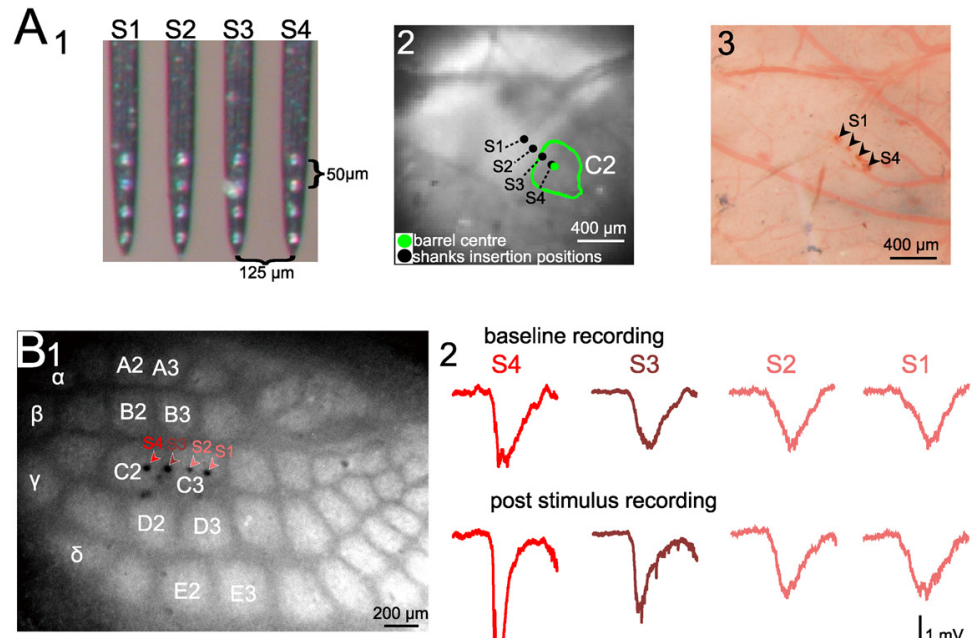

post stimulus recording
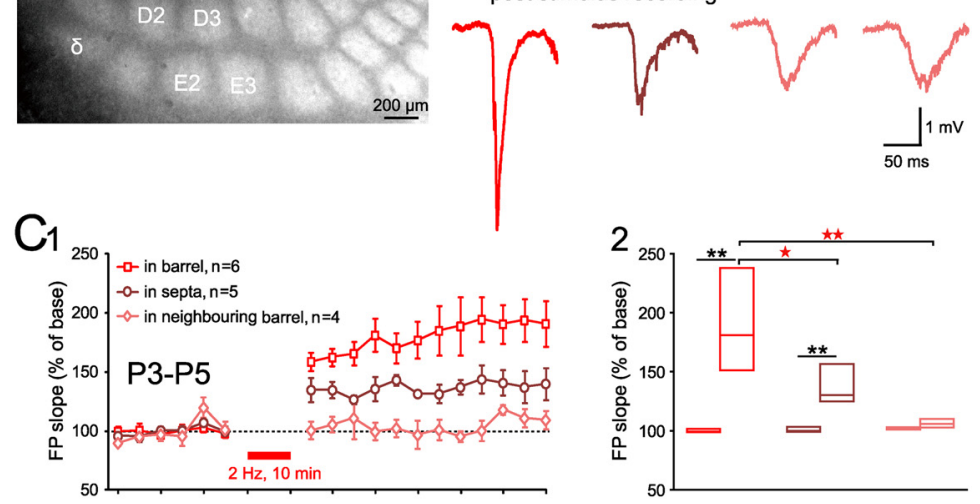

$\mathrm{D}_{1}$
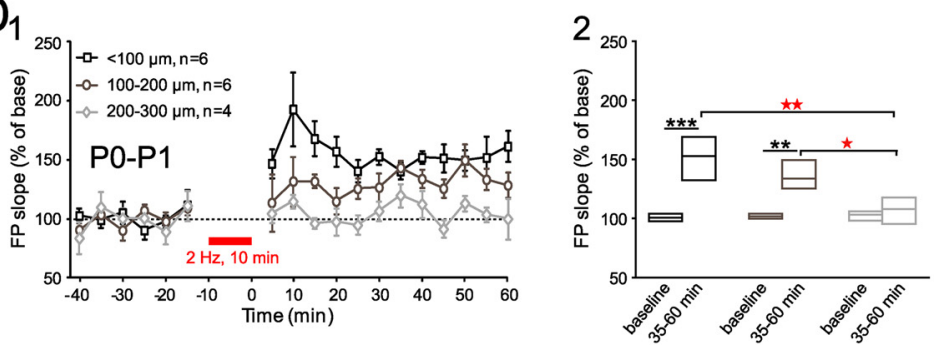

Figure 2. Spatial properties of LTP induced by repetitive single-whisker stimulation. $\boldsymbol{A}$, Photograph of the $4 \times 4$-channe Michigan electrode array $\left(\boldsymbol{A}_{1}\right)$. A local VSDI response was elicited by $\left(2\right.$ single-whisker deflection in a P4 rat $\left(\boldsymbol{A}_{2}\right)$. The green dot indicates the center of the $\mathrm{C} 2$ barrel-related cortical column. The black dots are the electrode insertion positions. The green circle represents the region of the $C 2$ whisker stimulation-evoked VSDI response. $A_{3}$ shows the same area as in $A_{2}$ after termination of the electrophysiological recordings and retraction of the recording electrode. The red dots and arrowheads show the electrode insertion points. $\boldsymbol{B}_{1}$, Photomicrograph of tangential section through layer IV of the barrel field of a P5 rat after processing the tissue for 5 -HTT staining. Colored arrowheads indicate different locations of the four shanks of the $4 \times 4$-channel Michigan electrode. $\boldsymbol{B}_{2}, \mathrm{FP}$ responses recorded in the activated barrel-related column, in the neighboring septum, and in an adjacent barrel during baseline and post-LTP induction periods from the same P5 rat as shown in $\boldsymbol{B}_{1} . \boldsymbol{C}_{1}$, Mean \pm SEM of relative FP responses recorded in the stimulated barrel (square), in the surrounding septal region (circle), and in the neighboring barrel (diamond) of P3-P5 rats. $\boldsymbol{C}_{2}$, Box plots of FP slopes recorded in barrel, septa, and neighboring barrel of P3-P5 rats averaged for baseline and 35- 60 min post-LTP induction. $D_{1}$, Mean \pm SEM of relative FP slopes recorded in various distances from the center of the activated barrel in $P 0-P 1$ rats. $D_{2}$, Box plots of FP slopes in various distances from the center of the stimulated barrel in P0-P1 rats averaged for baseline and 35- 60 min post-LTP induction.

visualized using a fluorescent antibody (Streptavidin Alexa-Cy3, 1:400, S-6402; Sigma). Slices were washed in PBS, mounted, and coverslipped.

Statistics. Data are presented as mean \pm SEM. In the bar diagrams, data are shown as box plots giving the median, 25th, and 75th percentiles; whiskers (10th and 90th percentiles); and outliers using SigmaPlot 10.0 software. Statistical analyses were performed with SPSS software version 13.0 using Mann-Whitney-Wilcoxon (for comparing two different groups), paired $t$ test (for comparing subsequent measurements in the same group of animals), and one-way ANOVA (for comparing more than two different groups) tests, followed by multiple comparisons with Bonferroni correction. Significance levels of $\left.p<0.001{ }^{(* *}\right), p<0.01$ $\left.{ }^{* *}\right)$, and $p<0.05\left(^{*}\right)$ were considered.

\section{Results}

Age-dependent expression of LTP in the neonatal rat barrel cortex in vivo

The FP responses to single-whisker stimulation recorded in newborn rat barrel cortex in vivo consisted of an early gamma activity followed by spindle bursts, as described previously (Minlebaev et al., 2007, 2011; Yang et al., 2009). To analyze activity-dependent modifications of the evoked responses, we quantified the slope of the initial negative-going FP response, which reflects the early activation of the cortex via the whisker-to-barrel cortex pathway.

In P3-P5 rats ( $n=16$ pups), repetitive single-whisker stimulation at $2 \mathrm{~Hz}$ for 10 min induced a significant $(p<0.001)$ increase in the slope of the FP that persisted for at least $60 \mathrm{~min}$. The FP slope increased to an average of $208.1 \pm 14.0 \%$ (Fig. $1 B_{1} ; B_{2}, B_{3}$, red symbols). No significant changes in the FP slope could be observed in the age-matched control group ( $n=9$ pups), which did not receive repetitive $2 \mathrm{~Hz}$ whisker stimulations (Fig. $1 B_{1}$; $B_{2}, B_{3}$, open symbols). These data demonstrate for the first time that the cerebral cortex of P3-P5 rats shows a prominent LTP to physiological stimulation of the afferent pathway in vivo.

Previous in vitro studies have documented, in thalamocortical slices of newborn rats, that the magnitude of LTP in barrel cortex gradually decreases between P3 and P7 and that LTP cannot be induced after the first postnatal week (Crair and Malenka, 1995). To address the question of whether a similar age-dependent expression of LTP can be also observed in vivo to physiologically relevant afferent stimulation, we studied the expression of LTP in $\mathrm{P} 0-\mathrm{P} 1$ and $\mathrm{P} 6-\mathrm{P} 14$ rats. In $\mathrm{P} 0-\mathrm{P} 1$ animals ( $n=12$ pups), single-whisker stimulation at $2 \mathrm{~Hz}$ for $10 \mathrm{~min}$ induced a significant $(p<0.001)$ and stable LTP to $150.7 \pm 3.6 \%$ of the baseline FP responses (Fig. $1 B_{2}, B_{3}$, black symbols). In P6-P7 rats ( $n=10$ pups), single-whisker stimulation elicited an increase in the FP slope to $149.9 \pm 23.6 \%$ (Fig. $1 B_{2}, B_{3}$, blue symbols), which is, however, not significantly $(p=0.351)$ different from the baseline responses. In P8-P14 rats, no obvious changes in the FP slope $(105.3 \pm 5.6 \% ; n=5$ pups) could be observed (Fig. $1 B_{2}, B_{3}$, green symbols). LTP in $\mathrm{P} 0-\mathrm{P} 1$ and $\mathrm{P} 6-\mathrm{P} 7$ rats was significantly $(p=0.027$ and $p=$ 0.037 , respectively) smaller when compared with the P3-P5 group. In summary, these results indicate that LTP is limited to the critical period with highest magnitude at P3-P5.

\section{Spatial expression of LTP}

Next, we studied the question of whether the expression of LTP is restricted to the stimulated whisker-related cortical column or 

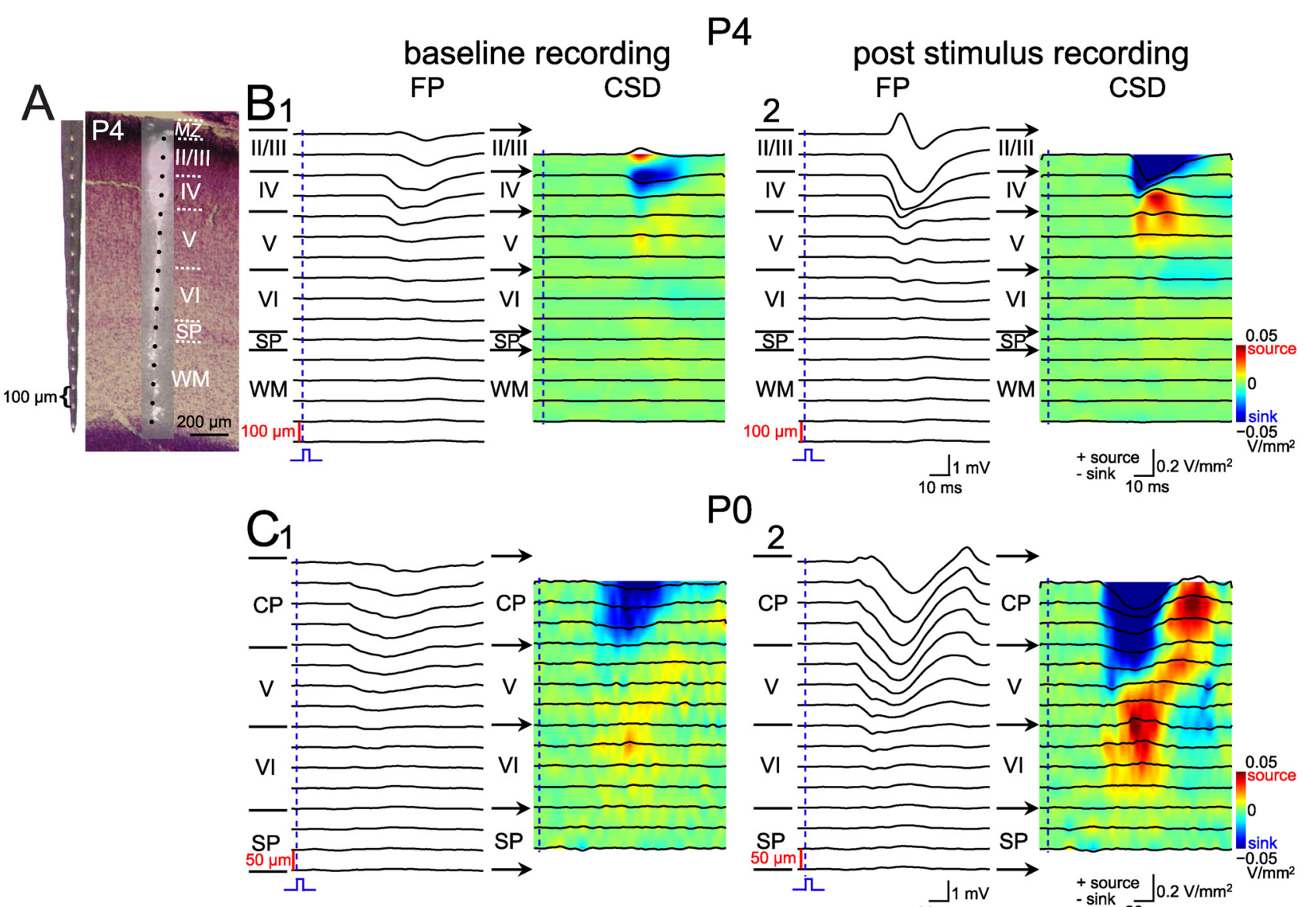

P0
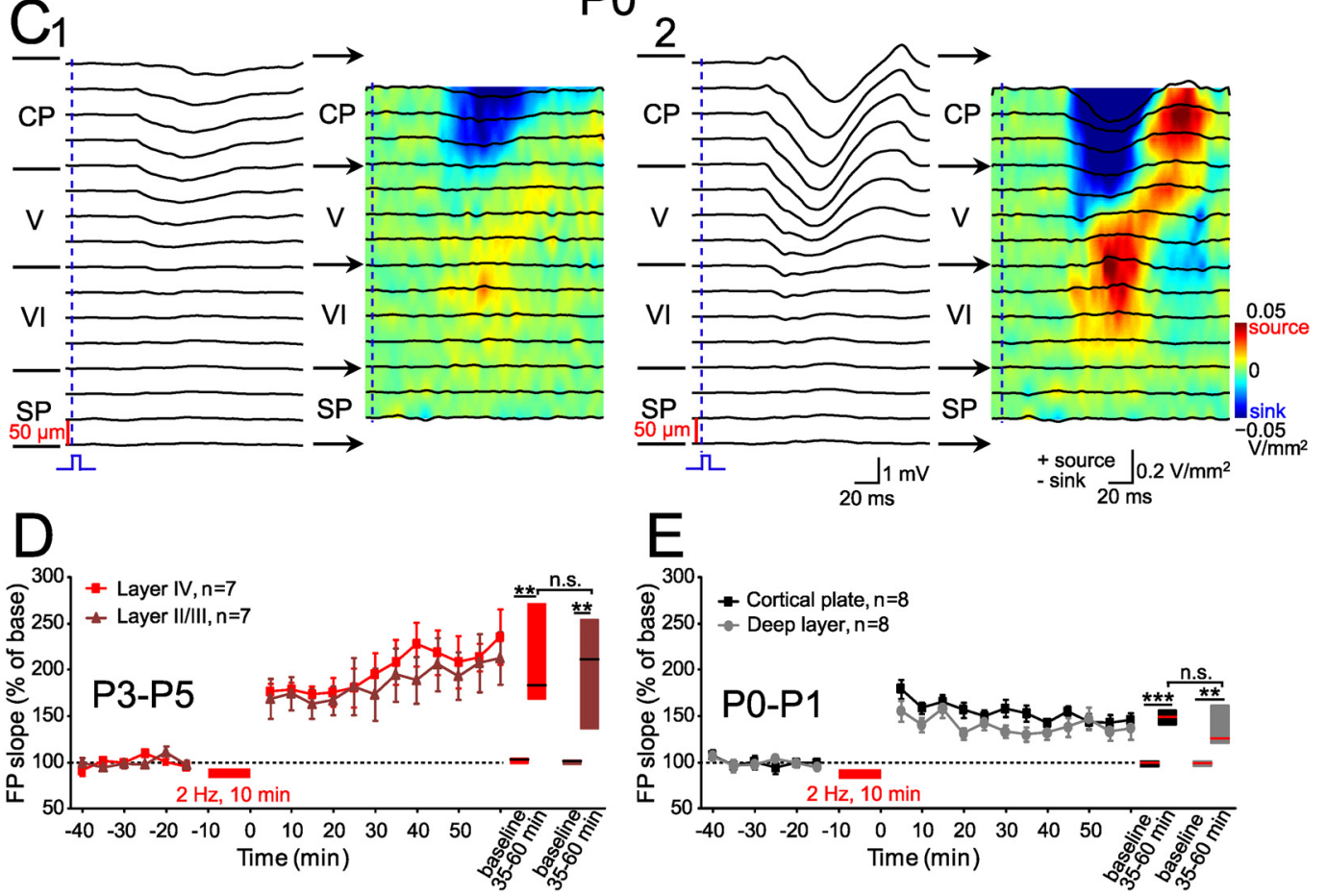

Figure 3. Representative FP response depth profiles and corresponding CSD analyses before and after induction of LTP in a P4 $(\boldsymbol{B})$ and PO $(\boldsymbol{C})$ rat. $\boldsymbol{A}$, photograph of the $1 \times 16$-channel Michigan electrode array (left). Digital photomontage reconstructing the location of the Dil-covered electrode in coronal Nissl-stained section (right). $B, C$, Depth profiles of FP responses to single-whisker stimulation and corresponding CSD analyses in a $\mathrm{P} 4$ and $\mathrm{P} 0$ rat. The interelectrode distance was $100 \mu \mathrm{m}$ in the P4 rat and $50 \mu \mathrm{m}$ in the P0 rat. $\boldsymbol{B}_{1}, \boldsymbol{C}_{1}$, Control data obtained during baseline recording. $\boldsymbol{B}_{2}, \boldsymbol{C}_{2}$, Data after LTP induction. In CSD analyses, current sinks (blue) are downward-going and current sources (red) are upward-going. $\boldsymbol{D}$, Relative FP slopes recorded in layer II/III and IV at P3-P5. $\boldsymbol{E}$, Relative FP slopes recorded in the cortical plate and deep layer at P0 $-\mathrm{P} 1$. Data are expressed as mean \pm SEM. Box plots represent FP slopes during baseline and 35- 60 min post-LTP induction (right). MZ, marginal zone; WM, white matter; SP, subplate; $C P$, cortical plate.

whether neighboring regions, septa and barrels, also show a significant LTP to our stimulation paradigm. Therefore, we inserted in the barrel cortex of P3-P5 rats a four-shank 16-channel Michigan-type electrode in the center of the whisker-related column as identified by VSDI (Fig. $2 \mathrm{~A}$ ). The horizontal distance of $375 \mu \mathrm{m}$ covered by these electrodes allowed the recording of FP responses in the activated barrel-related column and in neighboring regions, which, in all of these experiments, were unequivocally identified in post hoc 5-HTT staining of tangential sections through layer IV of the barrel cortex (Fig. $2 B_{1}$ ). Deflection of the C2 whisker induced characteristic FP responses in the whiskerrelated barrel, the septal region, and, with a smaller amplitude, in adjacent whisker-related barrels (Fig. $2 \mathrm{~B}_{2}$ ). Using this spatial information, the analyses demonstrated that the magnitude of LTP was largest in the barrel of the stimulated whisker ( $n=6$ pups), reaching an average value of $190.8 \pm 17.5 \%$ (Fig. $2 C$ ). In the septal regions, a significantly $(p=0.032)$ smaller LTP of $138.6 \pm$ $7.8 \%$ ( $n=5$ pups) was observed (Fig. $2 C$ ). No LTP could be observed in the neighboring nonstimulated barrel-related column ( $n=4$ pups).

No anatomical barrels could be observed in P0-P1 animals (Erzurumlu et al., 1990), but the spatial expression of LTP in this age group clearly correlated with the distance from the center of the whisker-related column, as identified from single-whisker stimulation-evoked VSDI responses (Fig. 2 $\mathrm{A}_{2}$ ). These experiments revealed a stable expression of LTP at electrode positions located $<100 \mu \mathrm{m}$ from the center of the barrel-related column (151.3 $\pm 7 \%, n=6$ pups; Fig. $2 D$ ). A lower LTP expression was 
observed at distances between 100 and $200 \mu \mathrm{m}(135.9 \pm 4.8 \%$, $n=6$ ), while no significant LTP could be observed at electrode distances $>200 \mu \mathrm{m}(n=4$; Fig. $2 D)$. In summary, these results suggest that the expression of LTP is mostly restricted to the activated barrel-related column already in newborn rat barrel cortex.

\section{Layer-specific expression of LTP}

To elucidate the laminar localization of the potentiated synapses, we performed CSD analyses in $\mathrm{P} 0-\mathrm{P} 1$ and $\mathrm{P} 3-\mathrm{P} 5$ rat barrel cortex in vivo. We compared the CSD profiles before and after the LTP induction in both age groups using FP recordings from oneshank 16-channel Michigan probes that covered all cortical layers from the cortical surface to the white matter (Fig. $3 A$ ).

In P3-P5 animals, the baseline CSD profiles were characterized by a prominent current sink in layer IV that extended into the lower part of layer II/III (Fig. $3 B_{1}$ ). These patterns are consistent with previously reported findings in the neonatal rat barrel cortex in vivo (Minlebaev et al., 2007; Yang et al., 2009). After 2 $\mathrm{Hz} 10$ min single-whisker stimulation, the synaptic potentiation was confined to this current sink (Fig. $3 B_{2}$ ), indicating a prominent increase in efficacy of the whisker-to-barrel cortex pathway innervating layer IV and lower layer II/III. Correspondingly, a stable LTP could be induced both in layer IV (219.2 $\pm 22.2 \%)$ and layer II/III $(200.8 \pm 26.6 \%)(n=7$ pups $)$. LTP amplitudes were not significantly $(p=0.655)$ different between these two layers (Fig. $3 D$ ). In $\mathrm{P} 0-\mathrm{P} 1$ animals ( $n=8$ pups), in which layers II/III and partly layer IV have not yet been formed (Molnár et al., 1998), a pronounced LTP consistent with synaptic potentiation of the current sink in the cortical plate $(147.5 \pm 3.2 \%, p<0.001)$ and in the deep layer $(136.6 \pm 7.6 \%, p<0.01)$ could be observed (Fig. $3 C)$. The LTP amplitudes were not significantly $(p=0.294)$ different between these two layers (Fig. 3E).

\section{Discussion}

We show here that physiologically relevant stimulation of the whisker-to-barrel cortex pathway induces in newborn rats in vivo a prominent LTP of the synaptic input that is confined to the activated barrel-related cortical column and adjacent septa. This LTP is strongest in P3-P5 rats, weaker in P0-P1 and P6-P7 rats, and disappears at $\mathrm{P} 8-\mathrm{P} 14$. These results demonstrate for the first time that the whisker-to-barrel cortex pathway in vivo may undergo activity-dependent modifications during earliest stages of postnatal development. However, as we did not record whiskerevoked synaptic responses in the trigeminal nuclei or thalamus, no conclusion about the exact site of synaptic potentiation can be drawn from these results.

Analyses of whisking behavior showed that adult rats actively sweep their whiskers over objects and surfaces to explore their environment, with a dominant frequency of $2 \mathrm{~Hz}$ (Carvell and Simons, 1990). However, rat pups do not perform active whisking during the first postnatal week, but receive physiological tactile stimuli at low frequency from interactions with their mother and littermates (for review, see Hanganu-Opatz, 2010). In the mature barrel cortex in vivo, rhythmic multiwhisker stimulation at $2 \mathrm{~Hz}$ could induce a stable LTP (Mégevand et al., 2009). These observations suggest that stimulation at $2 \mathrm{~Hz}$ might have some physiological correlates that are suitable to modify the synaptic plasticity in the whisker-to-barrel cortex pathway. Accordingly, we were able to potentiate this pathway with a $2 \mathrm{~Hz}$ single-whisker stimulation during the earliest postnatal development in vivo.

Our observations are in good agreement with previous in vitro reports on the age-dependent expression of LTP in the thalamo- cortical slice preparation of the newborn rat (Crair and Malenka, 1995). Crair and Malenka (1995) reported a gradual decrease in the magnitude of LTP from P3 to P7, while no LTP could be induced in P8-P14 animals. Our in vivo data fully support these previous in vitro findings, revealing a maximal magnitude of LTP between P3 and P5. In addition, we could demonstrate that LTP is weaker in P0-P1 animals and that no LTP could be induced at P8-P14, suggesting a transient period of enhanced synaptic plasticity that is limited to the critical period. Our data also indicate that the in vitro slice preparation is a valuable model to study activity-dependent modifications in immature cortex.

Our in vivo data also provide strong evidence that only synaptic inputs to the activated barrel-related column and, to a lesser extent, the surrounding septal region are modified. In P3-P7 rats, it can be assumed that our whisker stimulation protocol induces activity-dependent changes of synaptic inputs to layer IV and lower layer II/III (Fig. 3D) and our CSD data in the P3-P5 age group (Fig. $3 B$ ) are fully compatible with this hypothesis. The situation is different in P0-P1 animals, since layer II/III and, most likely, layer IV have not yet been formed at this early age. In somatosensory cortex of newborn rodents, the thalamic input predominantly innervates the subplate and is subsequently relayed to different cortical layers (for review, see Luhmann et al., 2009; Kanold and Luhmann, 2010). Accordingly in P0-P1 rats, an LTP was observed in both upper cortical layers and deep layers (Fig. 3E), which corresponds to the CSD data showing prominent synaptic inputs to these layers (Fig. 3C). However, no conclusive evidence for LTP of the thalamus-to-subplate synapse could be observed in this study (data not shown) or in thalamocortical slice preparations (our unpublished observations).

In conclusion, combining VSDI and multichannel electrode recordings in vivo, we demonstrate that single-whisker deflection at $2 \mathrm{~Hz}$ for $10 \mathrm{~min}$ induces an age-dependent LTP of the whiskerto-barrel cortex pathway in the corresponding barrel-related column and, to a lesser extent, in the adjacent septa. These activity-dependent modifications during the critical period may play an important role in the development and refinement of the topographic map in the barrel cortex.

\section{References}

Barth AL, Malenka RC (2001) NMDAR EPSC kinetics do not regulate the critical period for LTP at thalamocortical synapses. Nat Neurosci 4:235-236.

Berger T, Borgdorff A, Crochet S, Neubauer FB, Lefort S, Fauvet B, Ferezou I, Carleton A, Lüscher HR, Petersen CC (2007) Combined voltage and calcium epifluorescence imaging in vitro and in vivo reveals subthreshold and suprathreshold dynamics of mouse barrel cortex. J Neurophysiol 97:3751-3762.

Carvell GE, Simons DJ (1990) Biometric analyses of vibrissal tactile discrimination in the rat. J Neurosci 10:2638-2648.

Crair MC, Malenka RC (1995) A critical period for long-term potentiation at thalamocortical synapses. Nature 375:325-328.

Daw MI, Scott HL, Isaac JT (2007) Developmental synaptic plasticity at the thalamocortical input to barrel cortex: mechanisms and roles. Mol Cell Neurosci 34:493-502.

Erzurumlu RS, Jhaveri S, Benowitz LI (1990) Transient patterns of gap-43 expression during the formation of barrels in the rat somatosensory cortex. J Comp Neurol 292:443-456.

Feldman DE, Nicoll RA, Malenka RC (1999) Synaptic plasticity at thalamocortical synapses in developing rat somatosensory cortex: LTP, LTD, and silent synapses. J Neurobiol 41:92-101.

Foeller E, Feldman DE (2004) Synaptic basis for developmental plasticity in somatosensory cortex. Curr Opin Neurobiol 14:89-95.

Fox K (1992) A critical period for experience-dependent synaptic plasticity in rat barrel cortex. J Neurosci 12:1826-1838.

Fox K, Schlaggar BL, Glazewski S, O’Leary DD (1996) Glutamate receptor 
blockade at cortical synapses disrupts development of thalamocortical and columnar organization in somatosensory cortex. Proc Natl Acad Sci U S A 93:5584-5589.

Freeman JA, Nicholson C (1975) Experimental optimization of current source-density technique for anuran cerebellum. J Neurophysiol 38: 369-382.

Hanganu-Opatz IL (2010) Between molecules and experience: role of early patterns of coordinated activity for the development of cortical maps and sensory abilities. Brain Res Rev 64:160-176.

Inan M, Crair MC (2007) Development of cortical maps: perspectives from the barrel cortex. Neuroscientist 13:49-61.

Isaac JT, Crair MC, Nicoll RA, Malenka RC (1997) Silent synapses during development of thalamocortical inputs. Neuron 18:269-280.

Kanold PO, Luhmann HJ (2010) The subplate and early cortical circuits. Annu Rev Neurosci 33:23-48.

Krupa DJ, Brisben AJ, Nicolelis MA (2001) A multi-channel whisker stimulator for producing spatiotemporally complex tactile stimuli. J Neurosci Methods 104:199-208.

Luhmann HJ, Kilb W, Hanganu-Opatz IL (2009) Subplate cells: amplifiers of neuronal activity in the developing cerebral cortex. Front Neuroanat $3: 19$.

Mégevand P, Troncoso E, Quairiaux C, Muller D, Michel CM, Kiss JZ (2009)
Long-term plasticity in mouse sensorimotor circuits after rhythmic whisker stimulation. J Neurosci 29:5326-5335.

Minlebaev M, Ben-Ari Y, Khazipov R (2007) Network mechanisms of spindle-burst oscillations in the neonatal rat barrel cortex in vivo. J Neurophysiol 97:692-700.

Minlebaev M, Colonnese M, Tsintsadze T, Sirota A, Khazipov R (2011) Early gamma oscillations synchronize developing thalamus and cortex. Science 334:226-229.

Molnár Z, Adams R, Blakemore C (1998) Mechanisms underlying the early establishment of thalamocortical connections in the rat. J Neurosci 18:5723-5745.

Piñon MC, Jethwa A, Jacobs E, Campagnoni A, Molnár Z (2009) Dynamic integration of subplate neurons into the cortical barrel field circuitry during postnatal development in the Golli-tau-eGFP (GTE) mouse. J Physiol 587:1903-1915.

Takata N, Mishima T, Hisatsune C, Nagai T, Ebisui E, Mikoshiba K, Hirase H (2011) Astrocyte calcium signaling transforms cholinergic modulation to cortical plasticity in vivo. J Neurosci 31:18155-18165.

Yang JW, Hanganu-Opatz IL, Sun JJ, Luhmann HJ (2009) Three patterns of oscillatory activity differentially synchronize developing neocortical networks in vivo. J Neurosci 29:9011-9025. 